\title{
Organometallic vapor-phase homoepitaxy of gallium arsenide assisted by a downstream hydrogen afterglow plasma in the growth region
}

\author{
B. G. Pihlstrom, T. Y. Sheng, and L. R. Thompson \\ Colorado State University, Department of Electrical Engineering, Fort Collins, Colorado 80523 \\ G. J. Collins \\ Research Laboratory of Engineering Materials, Tokyo Institute of Technology, Yokohama 227, Japan
}

(Received 11 November 1991; accepted for publication 3 April 1992)

\begin{abstract}
In situ generated arsenic hydrides are reacted downstream with trimethylgallium (TMGa), both in the presence of and in the absence of a downstream hydrogen afterglow plasma. The homoepitaxial activation energy dramatically changes from $62 \mathrm{kcal} / \mathrm{mol}$ for the pure thermal to $21 \mathrm{kcal} / \mathrm{mol}$ for the plasma-assisted growth. The carbon incorporation mechanism for the plasma-assisted growth at temperatures less than $400^{\circ} \mathrm{C}$ has a distinct activation energy for carbon incorporation of $23 \mathrm{kcal} / \mathrm{mol}$, independent of V-III ratios. At temperatures above $400{ }^{\circ} \mathrm{C}$, the level of carbon incorporated in the films reaches a level that appears to be dependent on the gas-phase precursor V-III ratio. The activation energy of the low-temperature region is consistent with the surface decomposition of arsenic hydrides.
\end{abstract}

Heinecke et al. ${ }^{1}$ utilized a remote rf plasma to sensitize the arsine upstream from the introduction of the trimethylgallium (TMGa) for homoepitaxial GaAs. They found in direct comparison of the pure thermal to the plasmaassisted depositions that the growth activation energy decreased from 22 to $14 \mathrm{kcal} / \mathrm{mol}$. Huelman et al. ${ }^{2}$ found a comparable growth activation energy of $13 \mathrm{kcal} / \mathrm{mol}$ by remote plasma sensitizing the arsine. In pure thermal studies of Reep and Ghandhi ${ }^{3}$ the growth activation energy was found to be dependent on the TMGa-arsine gas-phase precursor ratios, varying from 16 to $20 \mathrm{kcal} / \mathrm{mol}$ as the V-III precursor ratios were varied from 36.9 to 9.3 , respectively. Thus, this implies a dependence of the growth rate on the relative concentration of reactive species of the hydride to the organometallic precursor.

Previous work by Tamaru ${ }^{4}$ has shown that the arsine decomposition is catalyzed by the presence of a surface and the resulting activation energy is $23 \mathrm{kcal} / \mathrm{mol}$. Carbon incorporation studies of the homoepitaxial GaAs growth by Reed et al. ${ }^{5}$ also exhibited an activation energy of $23 \mathrm{kcal} /$ mol. Reed postulated that the surface decomposition of the arsenic hydride species leads to the availability of accessible hydrogen which subsequently reacts with TMGa hydrocarbons to form volatile species.

Herein is described the study of GaAs homoepitaxial growth in the kinetically limited growth regime at substrate temperatures between 320 and $490^{\circ} \mathrm{C}$. We report on the behavior of the growth rate and carbon incorporation in an atypical growth region employing extremely low $\mathrm{V}$ III ratios $(<2.0)$.

The deposition procedure is as follows. A GaAs ( $\langle 100\rangle$ semi-insulating) substrate is loaded into the deposition reactor of Fig. 1 without any chemical degreasing or polishing. A turbomolecular pump creates a $10^{-6}$ Torr environment during pumpdown. The arsenic hydride utilized for the depositions is created via surface etching of solid arsenic by the hydrogen radicals generated in a microwave plasma. The arsine source is described elsewhere. ${ }^{6}$ The substrate is heated to $460{ }^{\circ} \mathrm{C}$ in the presence of the generated arsenic hydrides for 10 min prior to the growth. Following thermal preprocessing, GaAs deposition is initiated with the introduction of the TMGa either in the presence of or in the absence of the downstream hydrogen afterglow plasma. In either case, the arsine generator operating parameters are the same: $40 \mathrm{~W}$ microwave plasma power and $25 \mathrm{sccm}$ hydrogen flow. This plasma does not extend into the growth region. The $200-\mathrm{sccm}$ hydrogen flow shown in the top of Fig. 1 consists of molecular hydrogen when no active upstream plasma occurs. When the downstream hydrogen afterglow plasma is ignited, it provides to the growth region atomic hydrogen, hydrogen radicals, and vacuum ultraviolet (VUV) photons in addition to the molecular hydrogen. The VUV photons originate from the strong atomic hydrogen resonance lines (121.4 $\mathrm{nm}$ ). Its operating parameters are fixed at $300 \mathrm{~mA}$ at 600 $\mathrm{V} \mathrm{dc}$ bias $(180 \mathrm{~W})$. The epitaxial nature of all the deposited films discussed in Figs. 2 and 3 is verified via electron

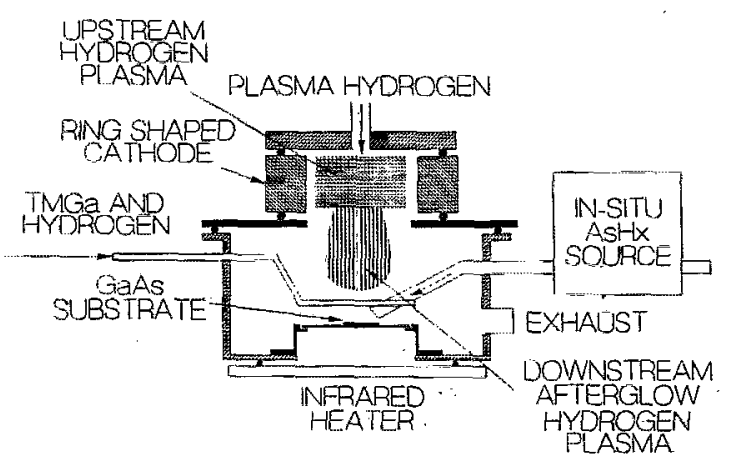

FIG. 1. Schematic representation of the homoepitaxial reactor employing in situ generated arsenic radicals and the presence or absence of an upstream hydrogen plasma. Ignition of the upstream hydrogen plasma creates a downstream afterglow in the growth region. Hydrogen flow rates are shown for both the arsenic hydride generation (bottom right) and for the upstream hydrogen plasma (top). The in situ arsine generator does not introduce a plasma to the growth region although it uses microwave excitation. 


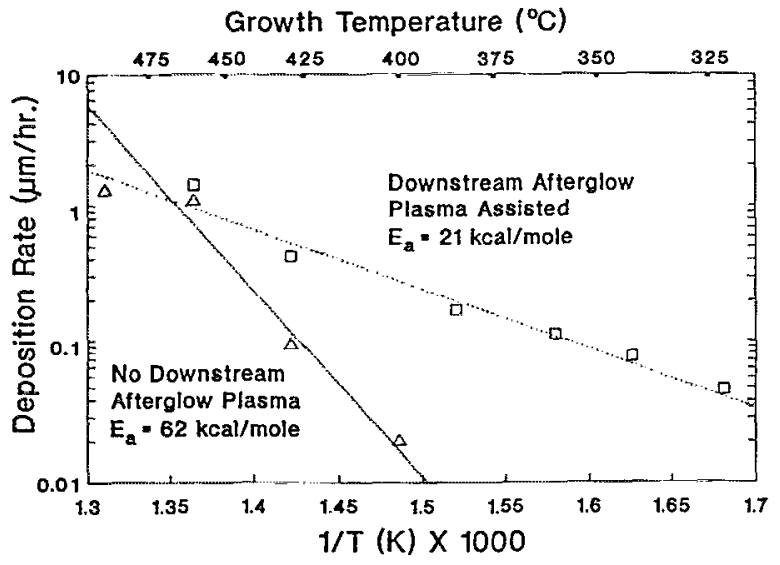

FIG. 2. Arrhenius plot of the homoepitaxial growth rate with and without the upstream hydrogen plasma ignited. The apparent activation energies are 62 and $21 \mathrm{kcal} / \mathrm{mol}$ for the thermal and the plasma-assisted cases, respectively.

channeling patterns in a scanning electron microscope (SEM) fitted with a backscatter detector. ${ }^{7}$

The homoepitaxial growth rate versus substrate temperature is shown in the Arrhenius plot of Fig. 2 for depositions with and without the upstream plasma. The data is from material that is deposited in layered structures in which constant reactor conditions (V-III ratios, total pressure, etc.) are maintained during growth while the substrate temperature is rapidly varied. Secondary-ion mass spectroscopy (SIMS) allows clear delineation of the material deposited at various substrate temperatures via the abrupt change in the carbon level. The overall film thickness is then measured with a stylus profilometer and related to the growth duration of each layer to achieve the growth rate at each deposition temperature. The activation energy observed for thermal growth, $62 \mathrm{kcal} / \mathrm{mol}$, is consistent with the heterogeneous decomposition of the TMGa, but is considerably higher than that normally seen for typical thermal organometallic chemical vapor deposition (OMCVD) growth using arsine.

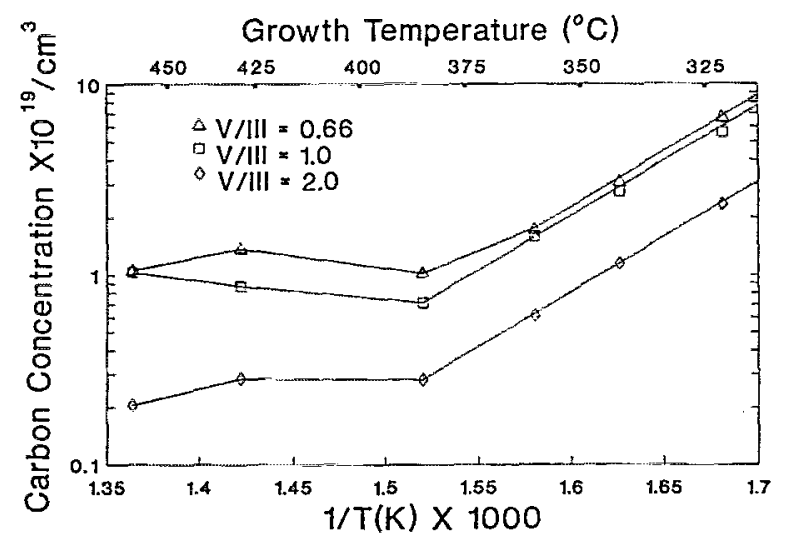

FIG. 3. Using plasma-assisted growth only we display the effect of varying V-III ratio on the carbon incorporation for various substrate temperatures employed.
Figure 3 plots the bimodal behavior of the carbon concentration in the GaAs films for the downstream plasmaassisted deposition as determined by argon-beam SIMS analysis. The concentration is plotted versus the reciprocal substrate temperature with V-III ratio variations of $0.66,1$, and 2 as an additional parameter. The absolute concentration was determined by calculating the ratio of the $\mathrm{As}^{75}$ to $\mathrm{C}^{12}$ positive secondary-ion counts/s in the deposited material and then comparing that to a $\mathrm{C}^{12}$-implanted GaAs standard.

The pure thermal growth activation energy of approximately $62 \mathrm{kcal} / \mathrm{mol}$ calculated from the data of Fig. 2 (e.g., no downstream hydrogen afterglow plasma) appears to be solely dependent on the heterogeneous decomposition of the TMGa precursor. Under identical conditions with the addition of the downstream hydrogen afterglow plasma, the growth activation energy is lowered from 62 to $21 \mathrm{kcal} / \mathrm{mol}$. The remote plasma has been shown to decompose TMGa at room temperatures, ${ }^{8}$ but the remote plasma effect may be more consistent with sensitizing the arsine.

The incorporation behavior of carbon shown in Fig. 3 has a distinct activation energy in the low-temperature region of $23 \mathrm{kcal} / \mathrm{mol}$ that appears to be independent of the limited V-III ratio explored. This coincides with the activation energy of Tamaru ${ }^{4}$ for the heterogeneous decomposition of arsine and is similar to the results of Reed et al. ${ }^{5}$ Reed concluded that the surface decomposition of arsenic hydrides creates accessible hydrogen which promoted the formation of volatile hydrocarbons, removing them from the growth interface.

The specific substrate temperature at which the carbon incorporation mechanism changes modes appears to closely coincide with the onset of thermal growth (no upstream afterglow plasma). However, the plasma-assisted growth activation energy appears to be constant through this transition, suggesting that the rate-limiting step in the deposition throughout the temperature range explored may be that of the surface decomposition of the arsine species. The accessible hydrogen generated would readily react with the hydrocarbon radicals at the growth interface to form stable species which would then be transported from the surface, allowing for the continuation of growth.

In conclusion, in situ generated arsenic hydrides are mixed with a TMGa precursor to achieve GaAs homoepitaxy either with or without a downstream afterglow hydrogen plasma. We have studied the effect of the downstream hydrogen plasma on both the homoepitaxial growth rate and carbon incorporation.

This work was supported by SERI No. XM-0-019142 (J. Benner) and NSF DMR Exploratory Research (Dr. L. Hess) and the National Science Foundation U.S.-Japan Research Corporation Program (NSF No. INT-9007937, Dr. Alex De Angelis). We would like to thank D. Kisker of IBM, Yorktown Heights, NY for his critical discussion of this work, Dr. McIntyre of Ford Microelectronics, Col- 
orado Springs, $\mathrm{CO}$ for the GaAs substrate donation, and $\mathrm{C}$. Messer and T. Potts of Hewlett Packard, Fort Collins, CO for the SEM backscattering analysis.

${ }^{1}$ H. Heinecke, A. Brauers, H. Luth, and P. Balk, J. Cryst. Growth 77, 241 (1986).

${ }^{2}$ A. D. Huelsman and R. Reif, J. Vac. Sci. Technol. A 7, 2554 (1989).

${ }^{3}$ D. H. Reep and S. K. Ghandhi, J. Electrochem. Soc. 130, 675 (1983).
${ }^{4}$ K. Tamaru, J. Phys. Chem. 54, 777 (1955).

${ }^{5}$ A. D. Reed, S. S. Bose, and G. E. Stillman, Appl. Phys, Lett. 54, 1262 (1989).

${ }^{6}$ B. G. Pihlstrom, T. Y. Sheng, L. R. Thompson, and G. J. Collins, J. Electron. Mater. 21, 277 (1992).

${ }^{7}$ D. Joy, D. Newbury, and D. Davidson, J. Appl. Phys. 53, R81 (1982).

${ }^{8}$ T. Y. Sheng, B. G. Pihtstrom, Z. Yu, and G. J. Collins, Appl. Phys. Lett. 54, 2411 (1989). 